\title{
Electrodynamics of Superconductors in Weak Fields
}

\author{
Amensisa Abdi \\ Department of Physics \\ University of Cincinnati \\ Cincinnati, Ohio 45221
}

Nov 28, 2001

\begin{abstract}
The discovery of superconductivity in a mercury sample by Heike Kamerlingh Onnes in 1911 have led to the rapid development and advancement of high $T_{c}$ superconductivity technology [1]. The highest $T_{c}$ of superconductors was that of the compound Nb3Ge until 1986 when two scientists J. G. Bednoz and K. A. Mller reported possible superconductivity in a mixture of $\mathrm{La}$ and $\mathrm{Ba}$ Copper Oxides at temperature of $30 \mathrm{~K} \mathrm{[2].} \mathrm{More} \mathrm{recently,} \mathrm{scientists} \mathrm{have} \mathrm{reported} \mathrm{superconductivity}$ at a temperature as high as $150 \mathrm{~K}$ in an oxide containing mercury [3]. However, to develop a new technology that utilizes superconductors, a complete understanding of the macroscopic electrodynamics of superconductors is important. Therefore, in this paper, some basic phenomena of superconductors and the macroscopic electrodynamic theory of superconductors in weak fields are discussed.
\end{abstract}




\section{Introduction}

The fascinating phenomena of superconductors and their potential applications had attracted not only experimentalists for finding new superconductors, but also attracted theoreticians in order to understand the physics behind superconductivity to describe the electrodynamics and thermodynamics properties of superconductivity. Intense research has been done toward this both on the microscopic and the macroscopic properties of superconductivity. In general, the microscopic properties of superconductivity are described by the well-known BCS theory which is developed by Bardeen, Cooper and Schriefier in 1957, where as the macroscopic behaviors of superconductivity are described by the well-known London theory developed by the brothers F. and H. London in 1937[4].

\section{Basic Phenomena of Superconductivity}

Conduction electrons that is free to move through the material carry the current in conductors. It is well known that electrons have a wave like nature and thus can be represented by a plane wave traveling in the same direction. The property of plane wave is that it can pass through a perfect crystal without being scattered since the atoms in a perfect crystal has a definite equilibrium location in regular array. That means, electrons can move through a perfect crystal without experiencing any resistance. However, actual crystal is never perfect due to defects like missing atoms, presence of impurity, atoms out of place, and thermal vibration of atoms. Therefore, plane wave moving in a crystal scatters. And electrons moving in materials experience resistance.

Generally, resistance of a metal or alloy decreases with temperature since the thermal vibration of atoms decrease. In 1911, Onnes found that the resistance of a mercury sample fell sharply at a temperature below $4.2 \mathrm{~K}$. Soon, this new phenomenon of zero dc-resistance at low temperature was found in may other metals. The temperature at which superconductivity first occurs is thus termed the superconducting transition (or critical) temperature of the material and denoted by $T_{c}$. The practical measurement of $T_{c}$ of a superconductor is usually made using three basic methods, method of resistance measurement, methods of magnetic-permeability measurement, and methods of specific heat measurement.

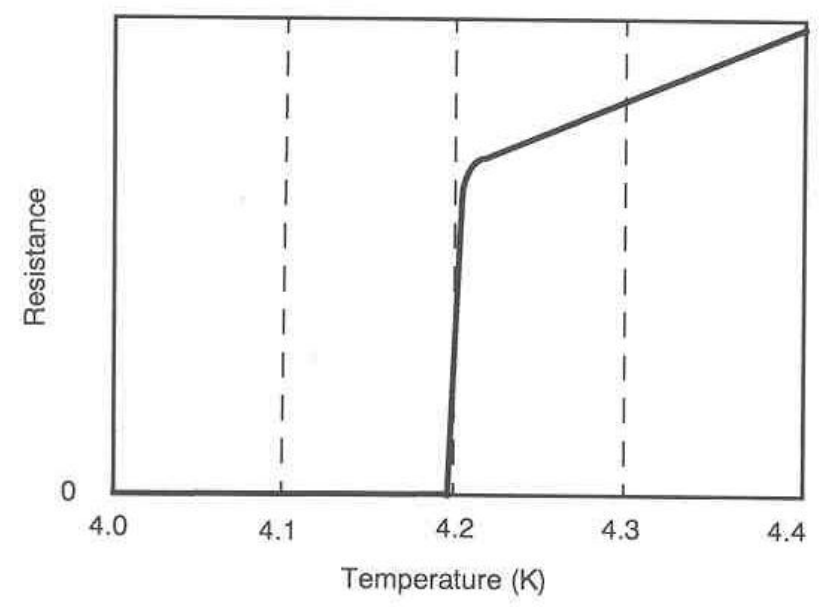

Figure 1: Resistance of a mercury sample at low temperature 
It has been found that the behavior of zero dc-resistance of superconductor is modified significantly at very high frequency of alternating current as shown by London in 1940[4]. The two-fluid electron model may explain this property of superconductors. According to two-fluid electron model, super electrons and normal electrons whose relative density depends on temperature carry current in superconductors. In dc case, current is total carried by superelectrons which does not need lectric field to move. In alternating field case, current in superconductors is carried by normal electrons in addition to superelectrons and thus resistance. When the frequency is in the order of $1013 \mathrm{~Hz}$, the resistance of superconductors would the same as the resistance of normal conductor independent of temperature. Onnes also found that the superconductivity could be destroyed when magnetic field is applied. The minimum magnetic field needed to destroy depends on the shape and orientation of the sample. When a cylindrical specimen is placed in a magnetic field in such a way that its axis is parallel the field, the transition is sharp and the minimum magnetic field needed to destroy superconductivity is called the critical magnetic field and denoted by Bc[1]. Within only small deviations, the following relation gives the temperature dependence of $\mathrm{Bc}$.

$$
B_{c}(T)=B_{c o}\left[1-\left(\frac{T}{T_{c}}\right)^{2}\right]
$$

Where $B_{c o}$ is critical field at zero temperature.

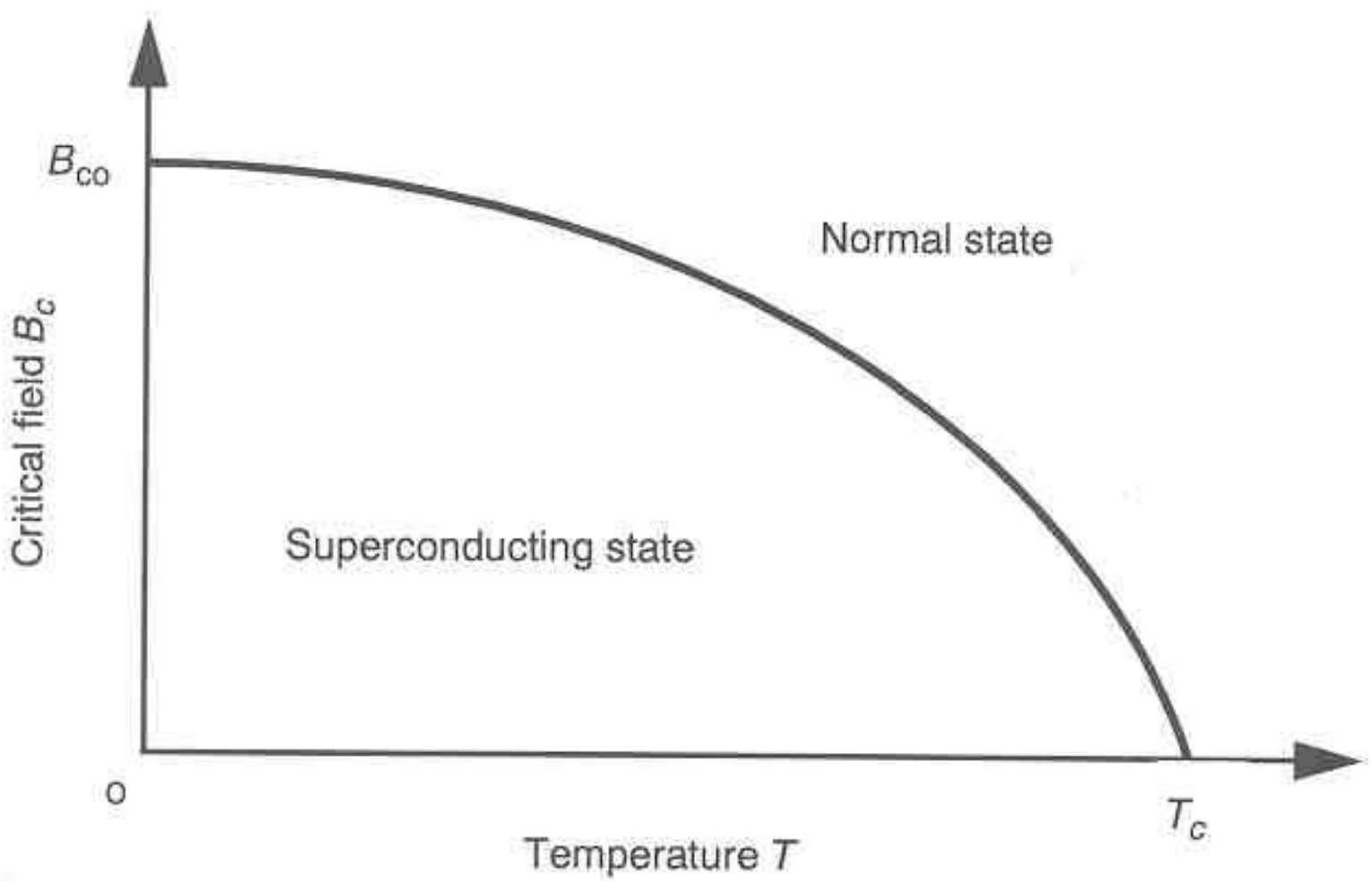

Figure 2: The critical magnetic field as a function of temperature

The absence of any magnetic flux in superconductivity is other fundamental property of superconductors (The Meissner Effect)[1]. Thus, superconductor behaves not only like perfect conductor of infinite conductivity but also like perfect diamagnetic material of $\mathrm{B}=0$. 
The infinite conductivity of superconductors is taken for grant to explain electrodynamics of superconductors till Meiser and Ochsenfeld (1933) measured the field surrounding a superconductor and concluded that the magnetic induction field B inside a superconductor is zero.

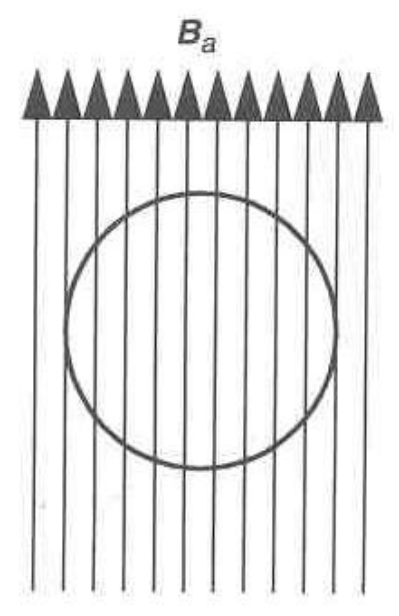

(a) Normal state $\left(T>T_{c}\right)$

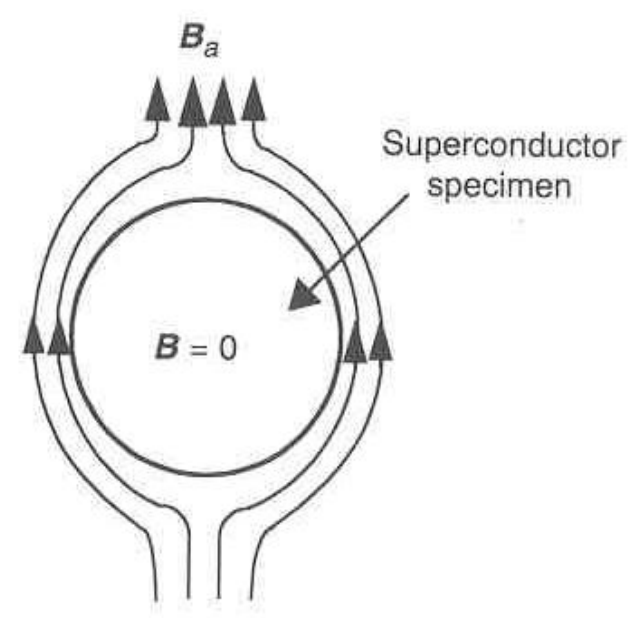

(b) Superconducting state $\left(T<T_{c}\right)$

Figure 3: The Meissner effect of a superconductor

\section{The London Theory of Superconductors}

Before the discovery of the Meissner effect in superconductors, the electrodynamic behaviors of superconductors is analyzed with the use of a simple free electron model, in which electrons accelerate without any resistance under applied electric field. E. Becker, Heller, and Sauter argued that if the electrons encounter no resistance, an applied electric filed would accelerate them steadily according to Newtons law [4]:

$$
m^{*} \frac{d v_{s}}{d t}=e^{*} E
$$

Where $\mathrm{m}^{*}$ and $\mathrm{e}^{*}$ are the effective mass and charge of the superelectrons respectively. The supercurrent density denoted by Js can be written in terms of the mean local velocity Vs as

$$
J_{s}=n_{s} e^{*} v_{s}
$$

Where $n_{s}$ is the superelectrons number density. Substituting eq. (3) into eq. (2) and taking linear approximation, we may obtain 


$$
\mu_{o} \lambda_{L}^{2} \frac{\partial J_{s}}{\partial t}=E
$$

Where $\lambda_{L}$ is a parameter of length dimension defined by

$$
\lambda_{L}=\sqrt{\frac{m^{*}}{\mu_{o} n_{s} e^{* 2}}}
$$

By taking the curl of both sides of eq. (4) and using Maxwell equations, we get

$$
\mu_{o} \lambda_{L}^{2} \frac{\partial}{\partial t}\left(\nabla \times J_{s}\right)=-\frac{\partial B}{\partial t}
$$

Using Maxwell equations and assuming any flux density in superconductors is due to current (no displacement current), we obtain

$$
\lambda_{L}^{2} \frac{\partial}{\partial t}\left[\nabla \times\left(\nabla \times J_{s}\right)\right]=-\frac{\partial B}{\partial t}
$$

Noting divergence of B is zero and using vector identity eq. (7) can be rewritten as:

$$
\lambda_{L}^{2} \nabla^{2} \frac{\partial B}{\partial t}=-\frac{\partial B}{\partial t}
$$

Substituting eq. (3.7) into eq. (3.5), we can obtain

$$
B=-\mu_{o} \lambda_{L}^{2} \nabla \times J_{s}
$$

The set of equations (4) and (9) describes the electrodynamic behaviors of superconductor according to London theory. Equation (4) is called the first London theory, describing the resistanceless properties of a superconductor, and equation (9) is called the second London equation, describing the Messner effect. Equation (9) can be also derived from more fundamental idea if superelectron fluid could be treated as a quantum mechanical quantity [4]. 


\section{Conclutions}

Superconductivity occurs in a metal or alloy below critical temperature. Superconductors are not only perfect conductors in which dc-resistance is zero but also they are perfect diamagnetic in which $\mathrm{B}$ field is zero. London electrodynamics theory developed by the brothers F. and H. London describes superconductors in week fields. That means London theory is in consistence with the experimental results zero dc-resistance and Meissner effect.

\section{References}

[1] Grosso, Giuseppe and Pastori Parravicini, Giuseppe, Solid State Physics (2000).

[2] J.G. Bednorz and K.A. Müller, Z. Phys. 64, 189(1986).

[3] A. Serway, Raymond and S. Faughn, Jerry, College Physics (1999)

[4] Zhou, Shu-Ang, Electrodynamics of Solids and Microwave Superconductivity (1999) 